\section{OC-006 INFLUENCE OF BMI AND ALCOHOL ON LIVER-RELATED MORBIDITY AND MORTALITY IN A COHORT OF 108,000 WOMEN FROM THE GENERAL POPULATION FROM UKCTOCS}

doi:10.1136/gutjnl-2013-304907.006

1."P M Trembling, ${ }^{2} \mathrm{~S}$ Apostolidou, ${ }^{3} \mathrm{~J}$ Parkes, ${ }^{2} \mathrm{~A}$ Ryan, ${ }^{2} \mathrm{~A}$ Gentry-Maharaj, 'S Tanwar, $2 \mathrm{U}$ Menon, 'W M Rosenberg. 'Institute for Liver and Digestive Health, Division of Medicine; ' ${ }^{2}$ ynaecological Cancer Research Centre, EGA Institute for Women's Health, University College London, London; ${ }^{3}$ Public Health Sciences \& Medical Statistics, Faculty of Medicine, University of Southampton, Southampton, UK

Introduction Alcohol and fat are major causes of chronic liver disease (CLD), however their relative influences are not well understood. We aimed to determine liver-related morbidity and mortality attributable to fat and alcohol by stratifying a cohort of 202,638 women according to BMI and alcohol intake.

Methods 107,742 women participating in the UK Collaborative Trial of Ovarian Cancer Screening where self-reported height, weight and alcohol intake were available were included. First episode related to cirrhosis (ICD-10 codes K70, K73, K74) either from inpatient Hospital Episode Statistics or death certificate was recorded following trial entry. Participants were stratified by low or high BMI ( $<25$ or $\geq 25 \mathrm{~kg} / \mathrm{m}^{2}$ ), low or high alcohol intake (0-15 or over 15 units/week) and combinations of these parameters.

Results Median age at recruitment was 60 years (50-75). Mean BMI was $26.4 \mathrm{~kg} / \mathrm{m}^{2}$. There were 90 events (54 inpatient episodes and 36 deaths). There was no difference in risk of event between the BMI groups, however there was a significant increase in risk in the high alcohol group (Log-Rank $<0.001$ ). Cox proportional hazards regression analysis (covariates age, smoking, BMI and alcohol intake), found that compared to the low BMI/low alcohol group, there was an incremental increase in risk of event in the high BMI/low alcohol, low BMI/high alcohol and high BMI/ high alcohol groups respectively. However, only the combination of high BMI and high alcohol reached significance (table and figure).

\section{Abstract 0C-006 Table}

\begin{tabular}{llll}
\hline Group & $\begin{array}{l}\text { Number of participants } \\
(\%)\end{array}$ & $\operatorname{Exp(B)/HR~(p)~}$ & $\begin{array}{l}\text { 95\% Confidence } \\
\text { intervals }\end{array}$ \\
\hline Low BMI/Low Alcohol & $46,011(41.9)$ & 1.0 & \\
High BMI/Low Alcohol & $58,432(53.2)$ & $1.1(0.65)$ & $0.7-1.8$ \\
Low BMI/High Alcohol & $2,683(2.4)$ & $2.4(0.11)$ & $0.8-6.9$ \\
High BMI/High Alcohol & $2,616(2.4)$ & $5.3(0.002)$ & $2.5-11.4$ \\
\hline
\end{tabular}

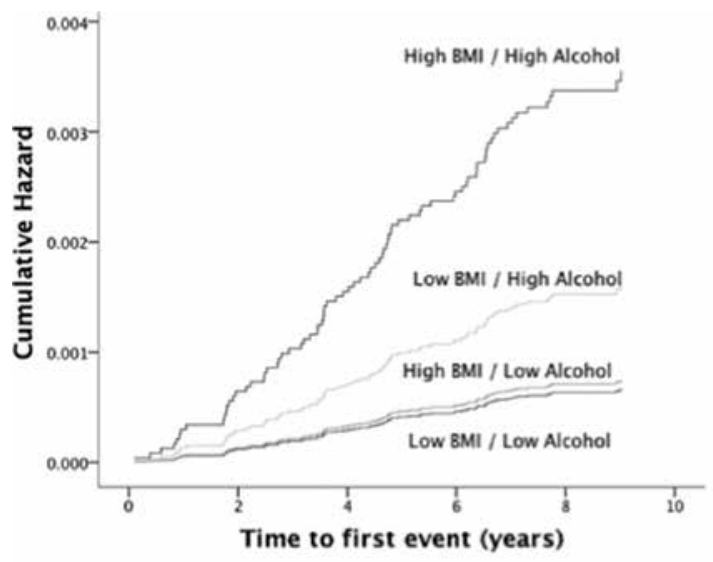

Abstract 0C-006 Figure
Conclusion These data indicate that the combination of high BMI and alcohol intake is associated with a synergistically increased risk of CLD. Alcohol may be the more significant contributing factor. Further work will define thresholds for each risk factor that independently and in combination increase CLD risk.

Disclosure of Interest None Declared

\section{OC-007 OXIDATIVE STRESS RATHER THAN TRIGLYCERIDE ACCUMULATION PERTURBS GLUTATHIONE METABOLISM IN AN IN VITRO MODEL OF CELLULAR STEATOSIS}

doi:10.1136/gutjnl-2013-304907.007

${ }^{1 .}{ }^{*} \mathrm{~K}$ A Lockman, ' L J Nelson, ${ }^{2} \mathrm{~J}$ R Manning, ${ }^{3} \mathrm{~K}$ E Burgess, ${ }^{4} \mathrm{~S}$ F Martin, ${ }^{4} \mathrm{~T}$ Le Bihan, ${ }^{2} \mathrm{D}$ R Dunbar, 'S D Morley, 'P C Hayes, 'I N Plevris. 'Hepatology; 'Bioinformatics Team, University/BHF Centre for Cardiovascular Science, University of Edinburgh, Edinburgh; ${ }^{3}$ Metabolomics Facility, University of Glasgow, Glasgow; ${ }^{4}$ Synthetic and Systems Biology, University of Edinburgh, Edinburgh, UK

Introduction Oxidative stress is the central to molecular events leading to the progression of simple steatosis to steatohepatitis in nonalcoholic fatty liver disease (NAFLD). We have previously shown that an in vitro cellular steatosis model using C3A cells treated with energy substrates; lactate $(\mathrm{L})$, pyruvate $(\mathrm{P})$, octanoate (O) and ammonia $(\mathrm{N})$, recapitulates the sequence of events in dietary-induced NAFLD; namely enhanced acute respiration and reactive oxygen species (ROS) formation leading to mitochondrial impairment. In contrast, treatment with oleate results in similar triglyceride accumulation but with relatively low ROS. Using a combined microarray, proteomic and metabolomic approach, we aimed to explore how triglyceride accumulation and enhanced ROS affect glutathione metabolism in our in vitro cell model.

Methods C3A cells were treated with either LPON or oleate for 72 hours. Microarray RNA expression was measured using Illumina ${ }^{\circledR}$ Whole Human Genome BeadChip H12 Microarray. For proteomics, peptides were analysed by liquid chromatography (LC) coupled mass spectrometry (MS) (Agilent HPLC/OrbitrapXL). Data were quantified label-free using Progenesis LC-MS and MASCOT. For metabolomics, LC separation was performed using hydrophilic interaction chromatography with a ZIC-HILIC. MS was performed using Orbitrap Exactive with HESI 2 probe. Raw LC/MS data were processed with XCMS Centwave and mzMatch.

Results LPON led to 2-fold downregulation of GCLC (encodes glutamate-cysteine ligase catalytic subunit, the rate limiting enzyme for glutathione synthesis) and upregulation of GPX1 and TXNDC12. Expression of GCLC and TXNDC12 was unchanged with oleate. Metabolomics confirmed that oxidised glutathione, glutathione disulfide, was higher in LPON- than oleate-treated cells. Among glutathione $S$-transferase genes, GSTA1 was unchanged with oleate but was upregulated by LPON (2.4-fold). Similarly, GSTT1, GSTK1 and GSTO1 were significantly increased by LPON. In contrast, MGST2 expression was higher in oleate than LPONtreated cells. Finally, proteomics showed that microsomal glutathione $S$-transferase 2 was downregulated by 2.5 -fold by LPON.

Conclusion Our data show that increased ROS formation rather than triglyceride accumulation alters glutathione metabolism. Such alterations may influence susceptibility to further insults, particularly those accelerating glutathione depletion, for example, paracetamol overdose. Disclosure of Interest None Declared

\section{OC-008 KUPFFER CELLS PLAY A DUAL PRO-INFLAMMATORY AND PROTECTIVE ROLE IN NON-ALCOHOLIC STEATOHEPATITIS}

doi:10.1136/gutjnl-2013-304907.008

${ }^{1} \mathrm{~T}$ Vo, 1." D Reid, ${ }^{2} \mathrm{~W}-\mathrm{K}$ Syn, ${ }^{1 P}$ Beck, ${ }^{1} \mathrm{D}$ Muruve, ${ }^{1,1,3 \mathrm{~B}}$ Eksteen. ${ }^{1}$ Snyder Institute for Chronic Diseases, University of Calgary, Calgary, Canada; 'Institute of Hepatology, London; ${ }^{3}$ Centre for Liver Research, NIHR Biomedical Research Unit, University of Birmingham, Birmingham, UK 